Explanations of this dormitory being a focus of infec. tion are:

1. Some of the sewage garden workers have been accustomed to sleep in it.

2. Most of the recent admissions sleep here, among whom there might be at times an undiagnosed case of enteric whose delirium was mistaken for insenity.

3. The presence of typhoid carrier or carriers. Five isolsted outbreaks have occurred in patients or attendants connected with it since January, 1905, the last of which was an attendant who died from typhoid in October, 1910. One of these cases, however, worked in the sewage garden.

Several of the remaining outbreaks show an association with rats. The Winterton division, where there are between 500 and 600 male and temsile patients, is about five minutes' walk from the main asylum. It is surprisingly free from flies at all times. The same cannot be said of rats, No. 3 female waxd in particular being infested; recently there were two rat-holes leading into it. The nurses have on many occasions seen rats ran into the drain.pipe trom the lavatory. The ward is a large one, with accommodation for 80 patients, about half of whom are epileptics. About 10 per cent. only are females under 30 years of age. Three separate outbreaks have occurred here since 1896-A. S., aged 23, notified October 1st, 1903; 3 cases in the summer and autumn of $1905 ; 1$ case, S. A. S., aged 24, who died October 30th, 1910. With the exception of a small, recently-built inflrmary, which is free from rats, No. 3 female ward is the nearest to the sewage garden-the distance being about 500 yardswhere there are pipes discharging the sewage from it and the other wards of this division of the asylum. The sewage garden appears to be a good breeding place for rats, as many as eighty being killed in it within a weok. There axe numerous rat-holes in it and the adjoining fields at the present moment.

In No. 2 female ward, at the main asylum, a consider. able distance from the above, a nurse aged 26 years was notified on November 26th, 1909. Following on her at intervals 4 new cases occurred in the same ward, the last in October, 1910.

During this period rats have been frequently seen in the ward, especially in the scallery. The lavatory accom. modation here is insufficient, but this is being remedied. There may be a typhoid carrier among the patients in this ward.

The wife of an attendant living in the village of Sedgefield, a mile from the asylum, took typhoid in the autumn of 1902. There were 3 cases of typhoid at the asylum then. Her husband had a terrier which was killing rats every day about the institution. The dog stayed in his house at night. This appears to have been an isolated case in the village.

Her husband, T. D., aged 35, was himself notified December 9th, 1907, five years afterwards. He had now two dogs which were ratting every day in the neighbourhood of the Isolation Hospital, outside which he had been employed for some time, and where there had been previously cases of typhoid. Both dogs were kept inside the house he lived in at night.

There were 4 cases of typhoid in 1906 on the male side of the main building, the first being an attendant stationed in No.' 10 ward, the infirmary, where he and others were nursed. Over 200 rats were killed here in the year 1906-7.

There were 5 cases in connexion with No. 7 female recoiving ward and No. 8 female adjoining it, both at the main asylum, in the summer and autumn of 1901. Rats were present in these wards at the time, and are still seen in both.

Concurrent with these female cases, W. A., a male, aged 50 years, was notified. He was employed at the time in one of the houses on the estate where there was a dog with which he killed rats daily.

It would be instruotive to learn if an association with rats can be traced in other asylums and institution's where sporadic outbreaks occur not otherwise explainable; \&lso, if unexplained cases are common among people possessing dogs who go ratting.

UNDER the will of the late Karl Beit of Hamburg, the Heidelberg Institute of Cancer Research will beneflt to the extent of $£ 5,000$. During the last twelve months the Institute has received donations amounting to over $£ 500$.
MYASTHENIA WITH GNLARGEMENT OF THE THYMUS GLAND.

BY J. ODERY SYMES, M.D., PHYSICIAN TO THE BRISTOL GENERAL HOBPITAL.

The following are notes of a case of myasthenia with onlargement of the thymus gland:

A governess, 21 years of age, was sent to the Bristol General Hospital under my care, by Dr. Manning of Wells, on November uniform good health, and there was no unfavourable family history.

On November 15th she complained of pain in the neck and headache, and was thought to be suffering from a cold. On the 18th she was conflned to bed with weakness in the legs. The left leg was affected before the right, and the weakness was such that she could not turn herself in bed or stand upright. There was difflculty in swallowing, and on November 22nd a tingling sensation in the hands, and pain and loss of power in both arms, her friends stating that " ghe was able to move them slowly but there was loss of strength in them." They also state that at this time "she did not feel a touch or a pinch." The pulse was rapid, and there .were occasional attacks of heart failure.

On admission to the hospital the patient was much exhausted. When she had somewhat recovered, her manner was hysterical she giggled and laughed, and said that she could not control herself. For these reasons a detailed examination of her nervous system was postponed. The temperature on admission was $97^{\circ} \mathrm{F}$., pulse 106 , respirations 24. There was no fever throughout her stay in hospital. She was menstruating.

There was loss of power in the trunk, arms, and legs. The wrists, elbows, knees, and ankles could be flexed and extended, and both upper and lower limbs could bevoluntarily raised from the bed. They could only be raised for a short period, and the patient could not lift any object from the bed. She could not stand even with a moderate amount of support, the knees giving way beneath her. The muscles were not wasted. Occsionally there was twitching of the fingers. The facial expression was peculiar, there being ptosis of both lids, most marked on the left side, and inability to completely close the eyes, even during sleep. There was no squint, but the ball of the eye rolled up under the upper lid, so that only the sclerotic showed between the lids. This was constant during sleep, but only happened occasionally during the day. Mastication and swallowing were difficult, and saliva collecting in the mouth and mucus in the trachea gave rise to distress. It was difficult to get the patient to talk, but no defect of speech was present. There was no stridor and no enlargement of tonsils. The patient complained of a sensation of coldness in the legs, and a feeling of constriction behind the manubrium sterni, especially on swallowing. Sensation to touch and pain was normal. There was tenderness over the left ovary. The deep reflexes in the arms were normal, the left knee-jerk was not obtained, the right was present, but feeble. A feeble flexor plantar response was present on both sides. The sphincters were unaffected ; the pupils reacted to light and accommodation. The heart and lungs were normal, and the pulse rapid. The urine contained no albumen or sugar, specific gravity 1030, acid. The striking feature of the case was the extreme lassitude and muscular weakness without wasting.

Thirty hours after admission, at 1 a.m., the patient became restless, dyspnoeic, and cyanosed. Strychnine and oxygen were administered, but she died suddenly at 1.45 .

The post-mortem examination was performed by Dr. EmrysRoberts, who reported that the body was well developed and covered with fat. The palate was high and arched, teeth absent, no lobes to ears.

The thymus gland showed both lobes to be enlarged, each being $4 \frac{1}{2}$ in. long by $1 \frac{1}{2}$ in. broad. No cysts. The thyroid gland was also somewhat enlarged. The heart and lungs were normal. The stomach was normal. The duodenum showed a number of dilated capillaries, and the same condition, together with several haemorrhagic areas, was present in the ileum The spleen was enlarged. The liver and pancreas showed capillary dilatations and minute haemorrhages.

Microscopic examination of the organs showed a considerable amount of altered blood pigment or débris in the liver and thymus gland, and more recent haemorrhages and dilatation of the capillaries, such as were also present in the intestinal walls. The thymus gland was normal, except that no Hassell's corpuscles could be found.

When first seen, this case suggested a diagnosis of hysteria. The mental condition, the complaint of tired. ness and weakness, the ptosis, difficulty of swallowing, and breathlessness, were all compatible with such a diagnosis. The sudden fatal termination and the post. mortem appearances, however, taken in conjunction with the facial appearance, the peculiar nature of the muscular weakness, and the absence of sensory phenomens and the unaltered reflexes, all negatived this diagnosis. Bulbar paralysis would not account for the ptosis, and the ordinary symptoms of a polioencephalitis were not present. A toxic polyneuritis or paralysis of Landry's 
type was suggested, but the paralysis was not of the asconding type; the limbs were not flacoid and helpless, the reflexes were not entirely abolished, and implica. tion of the cranial nerves at so early a date would be most unusual.

I am inclined, therefore, to regard the case as one of myasthenia gravis, accompanied by hypertrophy of the thymus.

The sense of muscular weakness and tiredness, the implication of the facial and fancial muscles, the absence of sensory symptoms, of altered reflexes, and of muscular wasting are characteristic. The only diffioulty that presents itself is the extreme rapidity of the progress of the disease, the whole period covered by the history being only thirteen days. The average duration of sixty cases colleoted by Campbell and Bramwell ${ }^{1}$ is one and a half years from the first appearance of symptoms, but in one fatal case the disease lasted only fourteen days, and in another only a month.

Possibly the fatal termination was hastened by the presence of the catamenia, for it has been noted that patients are generally worse at this period. A complete post-mortem examination was not allowed, and consequently no examination of the nervous system could be made, whilst owing to the patient's briet stay in the hospital the myasthenic electrical reaction was not tested.

Such pathological changes as were found at the postmortem examination were, however, confirmatory of the diagnosis of myasthenia gravis, for in a very large propor. tion of such cases there have been found hyperplasia of the thymus, also of the thyroid and the spleen, with dilated capillaries, haemorrhages, and blood débris, not only in these organs, but also in the liver, kidneye, and muscles. The muscles were not examined microscopically, but in the other organs no typical lymphorrhages wore found, only dilated and engorged capillaries.

With regard to the causation of the condition and its relationship to the status lymphaticus little can be said. Buzzard thinks the symptoms of myasthenia "are best explained by assuming the presence of some toxic agent which has a special influence on the protoplasmic (as opposed to the fibrillar) constituent of volantary muscle," and in this case the presence of numerous minute haemor. rhages in the gastro-intestinal tract is certainly very suggestive of an infective process. The relation of such an infective process to the thymic alteration is not clear. There is no reason to think that this patient had at any time previously saffered from an enlarged thymus, bat the death symptoms, asphyxia, and reflex cardiac failure are like those of status lymphaticus. We can only conjecture that the enlargement of the thymus and spleen were compensatory to some lymphoid exhaustion brought about by an acute toxaemia, probably of gastro.intestinal origin.

1 Brain, vol. Xxviii.

\section{THE ANTIHAEMOLYTIC ACTION OF AREENIC.} BY

JAMES A. GUNN, M.A., M.D., D.Sc , $\triangle N D$

WILERED J. FELTHAM, M.B., Cr.B.

(From the Pharmacology Laboratory, Edinburgh University.)

Is a former number of this Journal one of us (Gann British Medical Journal, July 18tb, 1908) has pointed out that very dilate solutions of arsenious acid exert on the red blood corpuscles in vitro a distinct protective action against haemolysis by hypotonic saline solutions. The conclusion was also drawn that arsenious acid is rapidly fixed to the red blood corpuscles, and the suggestion was made that an action of arsenic of this nature may, in part at least, explain the benefit which results from the medicinal administration of arsenic in blood diseases, especially in pernicious anaomia, on the ground that it may for a time protect the erythrocytes from destruction by physiological or pathological processes. Farther experiments have been made to determine whether this antibaemolytic action of arsenious acid is manifested by other compounds of arsenic and against haomolytic agents other than hypotonic solutions.

As it is probable that arsenious acid, after its absorption exists in the blood partly as sodium arsenite and partly as sodium arsenate, experiments were made with both these salts. Haemolytic agents of diverse nature were em. ployed-namely, distilled water, cyclamin, and sodium glycocholate.

The normal saline solution (N.S.) was in all experiments 0.85 per cent. of sodium chloride in distilled water. A 1 per cent. suspension of human blood corpuscles was obtained by adding $0.025 \mathrm{c.cm}$. of blood to $2.5 \mathrm{c.cm}$. of normal saline solution, and suspensions of this percentage were used throughout. In experiments in which washed corpuscles were used, the corpuscles were thrice centri. fuged with fresh saline and the final sediment made up to 2.5 c.cm. in the usaal way.

I. Sodium Arsenite.

(a) Protection of Corpuscles against Haemolysis by Distilled Water.

In each of two tabes, $A$ and $B$, was placed 0.5 c.cm. of a 1 per cent. suspension of unwashed corpuscles. Tc A was then added $0.50 \mathrm{~cm}$. of a solution of sodium chloride 1 in 20,000 in N.S.; and to B. $0.5 \mathrm{c.cm}$. of a solution of sodium arsenite 1 in 20,000 in N.S. They were allowed to stand at room temperature for fifteen minutes, whereupon $0.7 \mathrm{c.cm}$. of distilled water was added to each tube. This produced in four minutes almost complete haemolysis in A, bat only slight hremolysis in B. The tabes were allowed to stand at room tempera. tare for eighteen hours, when it was found that the corpuscles in A were completely haemolyzed, whereas in B there was still a considerable sediment of unhaemolyzed corpuscles. In this experiment the corpuscles in $B$ were suspended in normal saline solution, containing sodium arsenite in the proportion of 1 in 40,000. The corpuscles in A were suspended in a normal saline solution containing an additional quantity of sodium chloride in the proportion of 1 in 40,000, which was added to the saline in order to render the osmotic concentrations of the two solutions as nearly as possible the same. After the lapse of fifteen minutes the same amount of distilled water was added to each tube, and this produced a distinctly greater heemolytic effect on the control corpuscles than on those corpuscles which were under similar condi. tions, apart from the influence of sodinm arsenite.

The conditions and results of this experiment may be stated briefly in the following table. As other experi. ments to be described were performed in a similar manner, they will be detailed in tabular form only.

$$
\text { Tube A. TABLE I. Tube B. }
$$

$\begin{array}{lll}\text { (1) } 0.5 \text { c.cm. blood suspension } & \text { (1) } 0.5 \mathrm{c.cm} \text {. blood suspension }\end{array}$ (unwsshed corpuscles).

(2) $0.5 \mathrm{c.cm}$. sodium chloride 1 in 20,000 in N.8. (unwashed corpuscles).

(2) 0.5 c.cm. sodium arsenite 1 in 20,000 in N.S.

Stood for 15 minutes at room temperature.

No haemolysis.

(3) $0.7 \mathrm{c.cm}$. distilled water.

Result.

In 4 minutes almost complete haemolysis.

In 18 hours complete hremo lysis.

A series of similar experiments was made and repeated, in which the corpuscles were subjected to solutions of sodium arkenite varying from 1 in 20,000 to 1 in 200,000. The same qualitative effect was obtained in all, though the antihaemolytic $\in$ ffect of the more dilute solutions was less pronounced. No protective effect was detected with solutions more dilute than 1 in 200,000 .

Of course for such experiments it is necessary to add to the tabes not much more distilled water than is sufficient to haemolyze the control corpuscles, otherwise complete haemolysis will occur in both tabes.

\section{(b) Protection of Corpuscles against Haemolysis by} Cyclamin.

To ascertain whether sodium arsenite protects the corpuscles against haemolysis by saponin substances, experiments were made with cyclamin, which is one of the most active haemolytic agents of the saponin group. A strength of oyclamin solution convenient for the amount of cor. puscles taken was ascertained by preliminary experiments and found to be 1 in 100,000 in normal saline solution. 